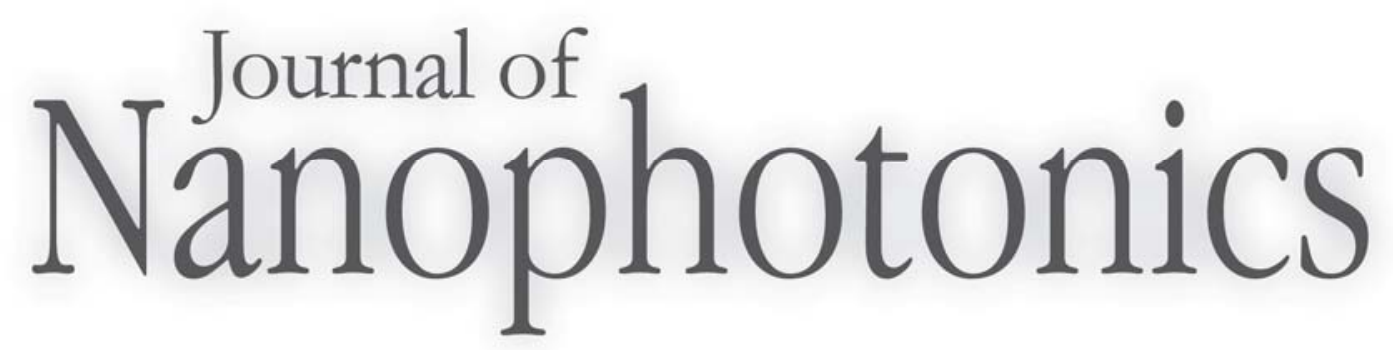

SPIEDigitalLibrary.org/jnp

\title{
Influence of silver clusters on the light absorption in $\mathrm{CdBr}_{2}-$ Ag crystals
}

Stefano Bellucci

Ivan Bolesta

Ivan Karbovnyk

Igor Kolych

Sergiy Martyniv

Sergiy Velgosh

\section{O SPIE}




\title{
Influence of silver clusters on the light absorption in $\mathrm{CdBr}_{2}$-Ag crystals
}

\author{
Stefano Bellucci, a Ivan Bolesta, ' Ivan Karbovnyk, ${ }^{\text {b }}$ Igor Kolych, b \\ Sergiy Martyniv, ${ }^{b}$ and Sergiy Velgosh ${ }^{b}$ \\ ${ }^{a}$ Istituto Nazionale di Fisica Nucleare, Laboratori Nazionali di Frascati, Via E. Fermi, 40, \\ 00044 - Frascati (RM), Italy \\ bellucci@lnf.infn.it \\ ${ }^{\mathrm{b}}$ National University of Lviv, Department of Electronics, 107 Tarnavskogo St., Lviv \\ 79017,Ukraine \\ bolesta@electronics.wups.lviv.ua
}

\begin{abstract}
Experimental studies of the absorption spectra of $\mathrm{CdBr}_{2}-\mathrm{Ag}$ photochromic crystals revealed several absorption bands positioned close to the intrinsic absorption edge. It is suggested that impurity silver ions, neutral silver atoms and intrinsic lattice defects form nanoclusters which are responsible for the absorption peaks within the transparency range of the crystal. The effect of UV radiation on the absorption profile is also discussed in the framework of the proposed model.
\end{abstract}

Keywords: clusters, absorption spectra, layered crystals.

\section{INTRODUCTION}

Cadmium bromide is a layered crystal having fundamental energy gap of $5.4 \mathrm{eV}$ [1]. Representing the wide group of divalent metal halides, $\mathrm{CdBr}_{2}$ has been thoroughly studied by optical spectroscopy techniques [2,3]. Particular attention has been paid to explain the photochromism effect in pure and doped $\mathrm{CdBr}_{2}$ [4-6].

Doping by impurities of different nature in order to "ensure" the required features of the material has long been a typical approach for the modification of optical-luminescent properties of solids. In the recent years, it became common to modify the properties of solids by introducing nano- or submicron sized clusters in the matrix of the given crystalline material. Clusters may contain not only the impurity atoms but as well lattice defects and/or matrix atoms. Being, in fact, quantum systems, nanoclusters possess discrete energy spectra and some of the energy states may be found in the band gap of the host material. Consequently, this influences the optical absorption, luminescence and photochromic processes in the investigated crystals.

It was proved that in copper doped cadmium bromide $\mathrm{Cu}^{+}$ions substitute $\mathrm{Cd}^{2+}$ at regular lattice sites. Induced negative effective charge is compensated by an interstitial copper ion $\left(\mathrm{Cu}_{i}^{+}\right)$. As a result $\left[\mathrm{Cu}^{+} \mathrm{Cd}_{-} \mathrm{Cu}^{+}{ }_{i}\right]$ centers are created. It is assumed that photochromic properties of $\mathrm{CdBr}_{2}-\mathrm{Cu}$ crystals are directly related to the charge state change of these centers, however being as well dependent on the concentration of other impurities [6].

Unlike $\mathrm{CdBr}_{2}-\mathrm{Cu}$ crystals, silver doped cadmium bromide has not been studied that much so far. Although $\mathrm{Ag}^{+}$ions (electronic configuration $4 d^{10}$ ) are homologically identical to $\mathrm{Cu}^{+}$, some differences in the absorption properties and in the nature of photochromism for this material may be expected which were not investigated in details [7].

In the present report we analyze the optical properties of $\mathrm{CdBr}_{2}-\mathrm{Ag}$ crystals with a particular focus on the role of clusters in absorption processes. 


\section{SAMPLES PREPARATION}

Bridgman-Stockbarger technique was employed to obtain $\mathrm{CdBr}_{2}-\mathrm{Ag}^{+}$crystals. Sealed quartz ampoules were used to provide the control of stochiometry. Silver was introduced by adding fixed amount of $\mathrm{AgCl}$ salt to the initial raw material. The samples for measurements were then cut along the cleavage planes. Linear dimensions of the prepared rectangular specimens were nearly $5 \times 5 \mathrm{~mm}$. Several samples of different thickness were cut in order to select the best absorption layer thickness, thus ensuring the good quality of the absorption measurements.

$\mathrm{CdBr}_{2}-\mathrm{Ag}^{+}$were colored by UV irradiation (DKSS-1000 xenon lamp combined with UFS-2 filter was exploited). After 3 minutes of irradiation stable colored samples were obtained. The bleaching of crystals was achieved by heating up to $T>600 \mathrm{~K}$.

Spectral measurements were performed with the equipment previously described in [8].

\section{RESULTS AND DISCUSSION}

Absorption spectra recorded for cadmium bromide crystals containing 0.1 molar percent of a silver salt in the melt are depicted in Fig. 1. The transparency region contains several absorption bands which are positioned close to the intrinsic absorption edge of $\mathrm{CdBr}_{2}$. Edge position is, in turn, in good agreement with exitonic absorption at $77 \mathrm{~K}$ reported in [9].

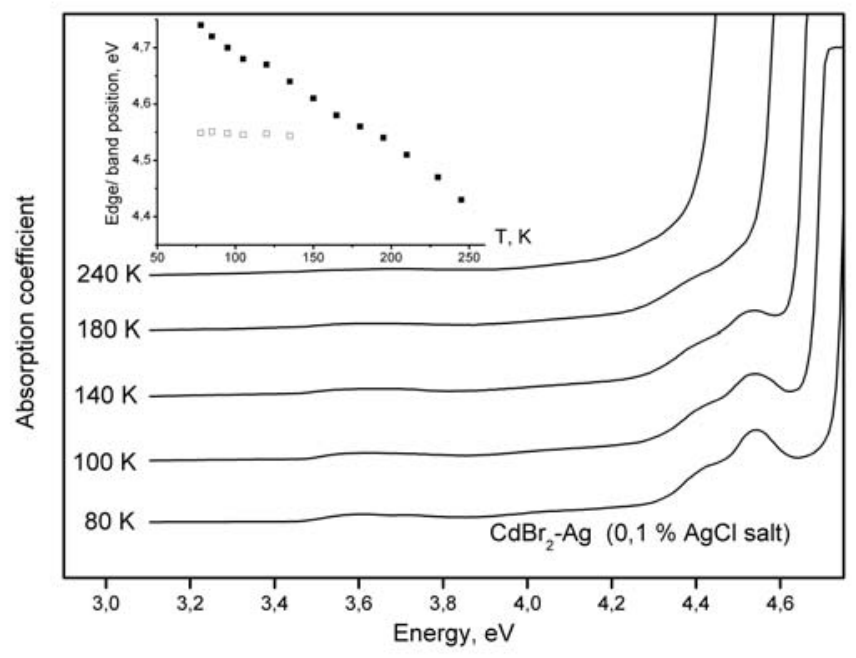

Fig. 1. Temperature evolution of the $\mathrm{CdBr}_{2}-\mathrm{Ag}(0.1 \% \mathrm{AgCl}$ salt added to the initial raw material) absorption spectra. Each spectrum is shifted with respect to the previous one for the purpose of comparison. Inset represents the temperature dependencies of the intrinsic absorption edge position and $4.54 \mathrm{eV}$ band.

Data processing procedure allowed to determine exact positions of the absorption maxima. Thus, the principal bands are peaked at $4.54 \mathrm{eV}$ and $4.43 \mathrm{eV}$, less intensive band backgrounded by strong absorption appears between $4.28 \ldots 4.00 \mathrm{eV}$ and weak peaks are observed also at $3.76 \mathrm{eV}$ and $3.60 \mathrm{eV}$.

The intrinsic absorption edge temperature dependence (see the inset in Fig. 1, filled squares) is close to linear. While the intrinsic absorption edge shifts at least for $0.3 \mathrm{eV}$ towards lower energies upon heating up to room temperature other abovementioned bands exhibit negligible dependence on temperature (for example, the $4.54 \mathrm{eV}$ band position is represented in the inset of the Fig. 1 by open squares). However, above $\sim 170 \mathrm{~K}$ their intensities are below the detectable level for the experimental equipment used. The band at 
$4.54 \mathrm{eV}$ obviously vanishes due to overlapping with edge absorption but there should be another reason for diminishing of three other bands, whereas at $170 \mathrm{~K}$ these band are peaked essentially below the absorption edge.

The absorption spectra are affected by UV-irradiation, as demonstrated in Fig 2.

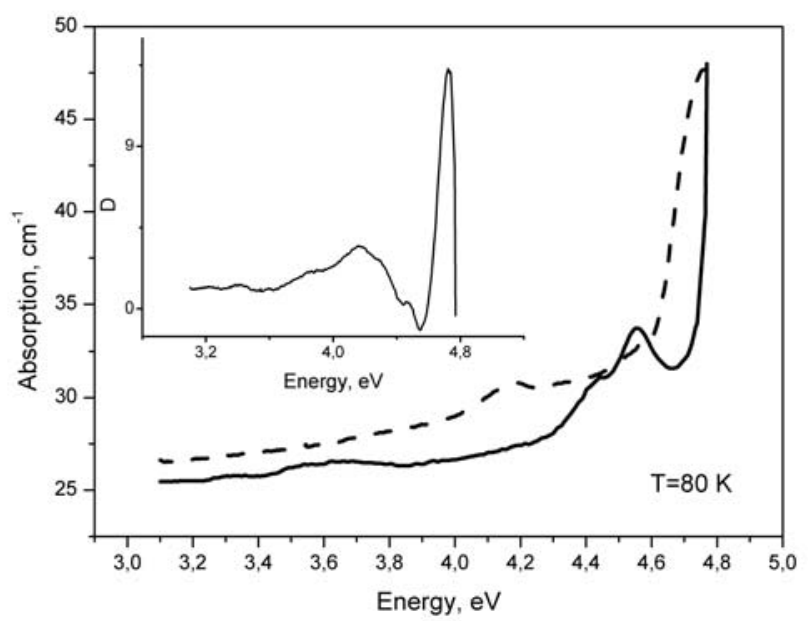

Fig. 2. Absorption spectra of $\mathrm{CdBr}_{2}-\mathrm{Ag}$ crystal at $80 \mathrm{~K}$ before (solid line) and after (dashed line) UV irradiation. Inset shows the difference between spectra of asgrown and irradiated crystals.

The influence of UV radiation is reflected in the shift of the absorption edge to lower energies. Analysing the difference spectrum one can conclude that such shift is due to the induced additional absorption around $4.68 \mathrm{eV}$. Besides, after the irradiation the intensity of the $4.43 \mathrm{eV}$ band decreases and significant background absorption is observed in the long wavelength region.

Performed measurements revealed complex character of the absorption spectra both for as-grown and $\mathrm{UV}$-irradiated $\mathrm{Ag}$-doped $\mathrm{CdBr}_{2}$ crystals. The band around $4.54 \mathrm{eV}$ is characteristic for the $\mathrm{CdBr}_{2}$ lattice and usually is tentatively ascribed to the intrinsic lattice defects [10]. In turn, other observed bands, namely those at $4.43 \mathrm{eV}, 3.76 \mathrm{eV}, 3.60 \mathrm{eV}$ and also peaks within the range of 4.28 to $4.00 \mathrm{eV}$ might be caused by the presence of silver impurity.

Following the example of the Cu-doped crystals one might assume that $4.43 \mathrm{eV}$ absorption is due to inner transitions within $\left[\mathrm{Ag}^{+} \mathrm{Cd}-\mathrm{Ag}^{+}{ }_{i}\right]$ centers, which are formed by substitution of $\mathrm{Cd}^{2+}$ ions by silver cations at regular lattice sites. In this case, however, the following contradiction arises. Since the ionic radius of silver is larger than that of copper, the lattice influence on the electronic transitions $4 d^{10} \rightarrow 4 d^{9} 5 s^{1}$ must result in the red shift of the absorption band ascribed to $\left[\mathrm{Ag}^{+} \mathrm{Cd}_{-}-\mathrm{Ag}^{+}{ }_{i}\right]$ centers with respect to absorption by $\left[\mathrm{Cu}^{+} \mathrm{Cd}^{-}\right.$ $\mathrm{Cu}^{+}$] centers which is reported at $4.13 \mathrm{eV}$ [4]. Red shifted from $4.13 \mathrm{eV}$ are weak bands at 3.76 and $3.60 \mathrm{eV}$ but low intensities of this peaks confirms the low probability of $\left[\mathrm{Ag}^{+} \mathrm{Cd}\right.$ $\left.\mathrm{Ag}^{+}\right]$centers creation. Aiming to explain the origin of the stronger $4.43 \mathrm{eV}$ peak and other observed absorption features we are proposing a cluster model.

During crystal growth there is a certain probability of the formation of neutral silver atoms (denoted hereafter as $\mathrm{Ag}^{0}$ ) due to the chemical reduction $\mathrm{Ag}^{+}+e^{-} \rightarrow \mathrm{Ag}^{0}$ (an electron may be captured from uncontrolled impurity). Neutral atoms, interstitial silver ions and substituted ions $\mathrm{Ag}^{+} \mathrm{Cd}$ tend to form nanosized clusters if some driving force that can stimulate this process is present [11]. Such a driving force results from elastic, Coulomb and chemical 
interactions between the components. Nanometric clusters exhibit discrete energy spectra [12]. Therefore, in case some energy levels fall within the bang gap of $\mathrm{CdBr}_{2}$, they can be responsible for additional absorption bands as well as for the centers of radiative and nonradiative recombination. In this sense nanoclusters behave similarly to the activator impurities which also lead to the appearance of local levels in the band gap. However, the important difference here is that bands originating from impurity centers are temperature sensitive (due to lattice thermal expansion and electron-phonon interaction) and cluster-related bands are not expected to be temperature dependent (the structure of clusters is almost the same for the wide range of temperatures).

Concluding, the band at $4.43 \mathrm{eV}$ which exhibits no temperature dependence in the experiment may be ascribed to the presence of nanoclusters composed of silver atoms or/and ions and intrinsic lattice defects. This band disappears above $170 \mathrm{~K}$ probably due to the fact that bonding energy between cluster components is too small at higher temperatures and clusters are destroyed.

Analyzing the influence of UV radiation on the $\mathrm{CdBr}_{2}-\mathrm{Ag}$ absorption spectra one should notice that since the energy of incident UV light is within the range of intrinsic absorption for the crystal under study, free electrons and holes are generated. This, consequently, results in more efficient silver reduction and increases the probability of cluster charge state change due to ionization (of due to capturing of a free carrier by a cluster). Hence, UV irradiation may lead to the modification in cluster structure and stimulate the formation of new clusters with different energy spectra. Similar processes have been reported to take place in water solutions [13] where $\mathrm{Ag}^{+}$ions and silver neutral atoms under UV radiation form small clusters which show a tendency to agglomerate in larger structures. As a result, a family of so called "magic" clusters $\left(\mathrm{Ag}_{2}{ }^{+}, \mathrm{Ag}_{3}{ }^{2+}, \mathrm{Ag}_{4}{ }^{2+}, \mathrm{Ag}_{8}{ }^{2+}\right)$ is formed. $\mathrm{Ag}_{8}{ }^{2+}$ cluster corresponds to the UV induced absorption peak at $4.28 \mathrm{eV}$.

Further increase in the number of particles induces the formation of quasi-metallic centers with characteristic broad absorption band within $3.35 \ldots 3.54 \mathrm{eV}$. Taking this into account one can explain features in the difference spectrum (inset in Fig. 2) by the formation of small silver clusters consisting of different number of particles. Thermal annealing of crystals within the model discussed should lead to the decomposition of clusters at higher temperatures and this is in accordance with the bleaching observed at $T>600 \mathrm{~K}$.

The suggested approach predicts the increase of the cluster formation efficiency as the silver contamination in the samples grows. To verify this idea, cadmium bromide crystal containing $1 \mathrm{~mol}$. percent of silver was prepared. The absorption spectra of this crystal is demonstrated in Fig. 3. The graph proves higher silver content increases the absorption coefficient in the range of 4.2-4.7 eV for more than two orders of magnitude as compared to the data of Fig. 2. Moreover, additional absorption appears at longer wavelengths (see the inset in Fig. 3), again without sharp band profiles. These results can be explained by the formations of different Ag-clusters, absorbing in a wide range of visible spectral range. This explanation agrees with assumptions made above. 


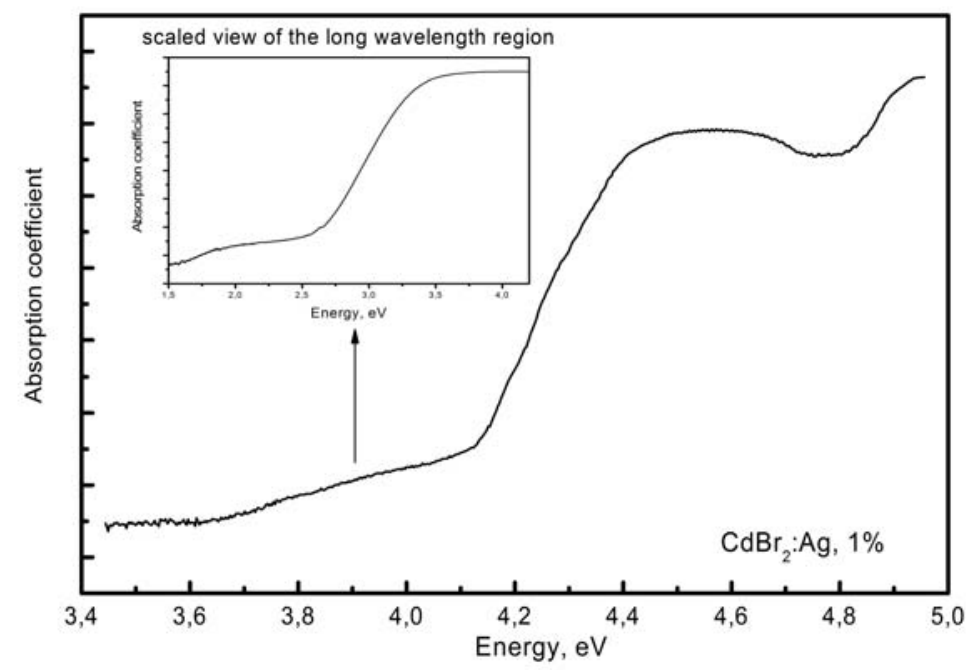

Fig. 3. Absorption spectra of $\mathrm{CdBr}_{2}-\mathrm{Ag}(1 \mathrm{~mol} \% \mathrm{Ag})$ at $T=78 \mathrm{~K}$. Inset shows enlarged graph of the long wavelength region of the spectrum.

In addition to indirect studies of clusters by means of optical spectroscopy, atomic force microscopy (AFM) was applied in order to analyze the distribution of silver in $\mathrm{CdBr}-\mathrm{Ag}$ crystalline samples. For this purpose Solver P47 PRO scanning probe microscope from NTMDT was exploited in phase imaging mode. The capabilities of the instrument combined with the enhanced contrast and sensitivity of the phase imaging allow in our case for detecting the variation in the composition at the nanoscale. Since, at low concentrations of silver in the investigated crystals there are certain difficulties in focusing the appropriate surface region by the probe, microscopy technique was applied to study $\mathrm{CdBr}_{2}$ with $1 \mathrm{~mol} \%$ of $\mathrm{Ag}$.

Fig. 4 shows the obtained phase contrast image of these crystals. The brief analysis allows to conclude that silver distribution is strongly nonuniform. Over the light gray background which corresponds to pure $\mathrm{CdBr}_{2}$ one can notice darker areas $\left(\mathrm{CdBr}_{2}-\mathrm{Ag}\right.$ phase with different silver content) and essentially dark regions which represent silver inclusions.

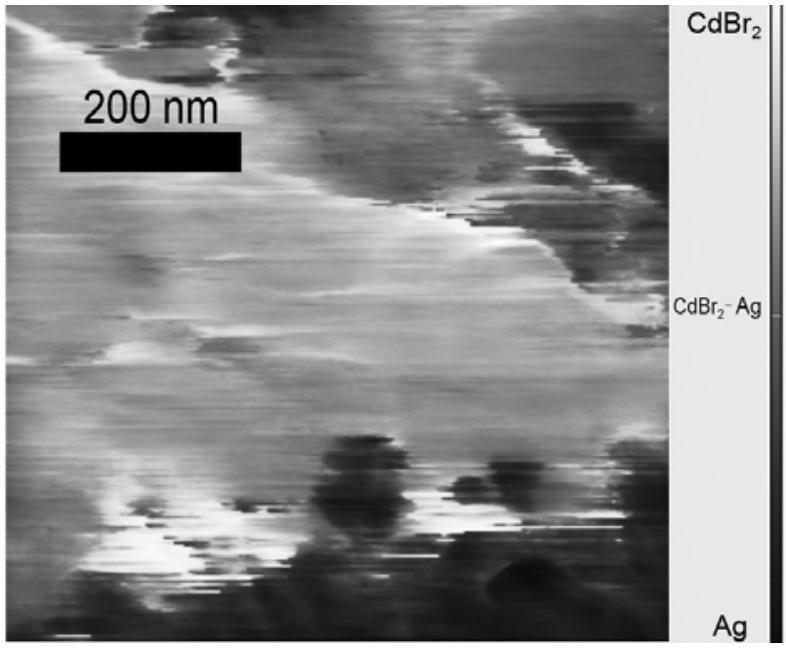

Fig. 4. Phase image evidencing the presence of silver clusters in $\mathrm{CdBr}_{2}-\mathrm{Ag}$ ( $1 \mathrm{~mol}$. $\% \mathrm{Ag}$ ) crystals. The bar on the right reflects the relation between the gradations of gray and the composition. 


\section{CONCLUSIONS}

Absorption spectra of cadmium bromide crystals doped with silver have been studied in the temperature range of $80 . .240 \mathrm{~K}$. Additional absorption bands within the transparency region for $\mathrm{CdBr}_{2}$ are assumed to be related with nanoclusters composed of silver impurity ions and lattice defects.

It is demonstrated that UV radiation induces the change in cluster charge state due to silver reduction reaction. UV stimulated absorption is ascribed to the $\mathrm{Ag}_{8}{ }^{2+}$ clusters and longwavelength features in the spectrum of irradiated $\mathrm{CdBr}_{2}$ are suggested to be due to quasimetallic centers, i. e. clusters, containing larger number of particles.

\section{References}

[1] I. Pollini, J. Thomas, R. Coehoorn, and C. Haas, "Optical reflectivity and electronic structure of layered cadmium halides," Phys. Rev. B 33, 5747-5755 (1986) [doi:10.1103/PhysRevB.33.5747].

[2] S. Kawabata and H. Nakagawa, "Life-time resolved emission spectra in $\mathrm{CdBr}_{2}$ crystals," J. Luminesc.112, 58-61 (2005) [doi:10.1016/j.jlumin.2004.09.083].

[3] K. Fukui, T. Kihara, and H. Nakagawa, "Luminescence and reflectance spectra of $\mathrm{CdBr}_{2}$ crystal," UVSOR Act Rep. 2000, 140 (2001).

[4] S. S. Novosad, I. S. Novosad, and A. V. Borodchuk, "Effect of iodine doping on the luminescent properties of the photochromic material $\mathrm{CdBr}^{2}: \mathrm{Cu}$," Inorg. Mater. 41, 187-190 (2005) [doi:10.1007/s10789-005-0041-0].

[5] H. Matsumoto, H. Nakagawa, and H. Kuwabara, "Optical properties of copper impurities in $\mathrm{CdCl}_{2}$ and $\mathrm{CdBr}_{2}, "$ J. Phys. Soc. Jpn. 44, 957-964 (1978) [doi:10.1143/JPSJ.44.957].

[6] S. S. Novosad, O. V. Gal'chynskyy, and R. O. Kovalyuk, "Effect of ultraviolet light irradiation on electrical properties of $\mathrm{CdBr}_{2}: \mathrm{Cu}^{+}$single crystals," Nucl. Instrum. Method. Phys. Res. B 116, 265-268 (1996) [doi:10.1016/0168-583X(96)00058-4].

[7] S. S. Novosad and B. M. Kostyuk, "Photostimulated processes in $\mathrm{CdBr}_{2}: \mathrm{Cu}, \mathrm{Pb}$ and $\mathrm{CdBr}_{2}: \mathrm{Ag}, \mathrm{Pb}$ crystals," Function. Mater. 5, 171-174 (1998).

[8] I. Bolesta, S. Velgosh, Yu. Datsiuk, I. Karbovnyk, V. Lesivtsiv, T. Kulay, A. I. Popov, S. Bellucci, M. Cestelli Guidi, A. Marcelli, and M. Piccinini, "Optical, infrared and electron-microscopy studies of $\left(\mathrm{Cd}_{i}\right)_{n}$ metallic clusters in layered $\mathrm{CdI}_{2}$ crystals," Radiat. Measure. 42, 851-854 (2007) [doi:10.1016/j.radmeas.2007.02.047].

[9] M. Kitaura and H. Nakagawa, "Polarization of self-trapped exciton luminescence in $\mathrm{CdBr}_{2}$," J. Luminescence 66-67, 438-442 (1996) [doi:10.1016/0022-2313(95)00186-7].

[10] A. B. Lyskovych, N. K. Gloskovska, and I. M. Bolesta. Wide Band-gap Layered Crystals and their Physical Properties, Vyscha Schkola, Lviv (1978) (in Russian).

[11] W. A. De Heer, "The physics of simple metal clusters: experimental aspects and simple models," Rev. Mod. Phys. 65, 611-676 (1993) [doi:10.1103/RevModPhys.65.611].

[12] J. P. Wilcoxon and G. A. Samara, "Strong quantum-size effects in a layered semiconductor: $\mathrm{MoS}_{2}$ nanoclusters," Phys. Rev. B 51, $7299-7302$ (1995) [doi:10.1103/PhysRevB.51.7299].

[13] B. G. Ershov and A. V. Gordeev, "Silver nanoparticles stabilised with heteropoly anions in an aqueous solution: optical properties and electronic polarisation," Mendeleev Commun. 11, 147-148 [doi:10.1070/MC2001v011n04ABEH001462]. 